\title{
Informationen aus Österreich
}

Manuelle Medizin 2018 · 56:116

https://doi.org/10.1007/s00337-0180370-0

(c) Springer Medizin Verlag $\mathrm{GmbH}$, ein Teil von Springer Nature 2018

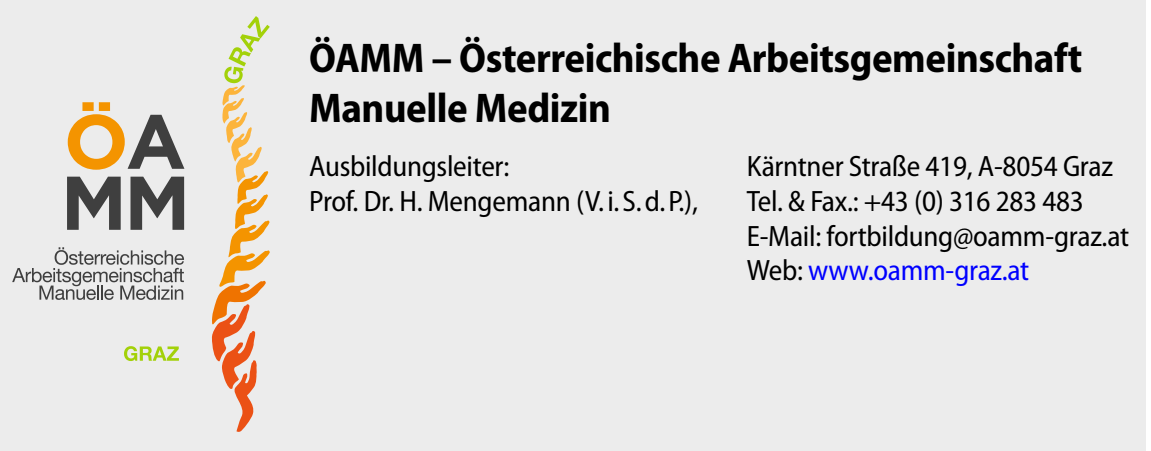

\section{Kurse 2018}

\section{Kurs 1}

21.06.-24.06./28.06.-01.07. Bad Häring

22.09.-29.09.2018

Graz

KA1813

KA1814

\section{Kurs 2}

29.04.-06.05.2018

09.06.-16.06. 2018

Wien

11.10.-14. 10./18.10.-21.10. Bad Häring

\section{Kurs 3}

01.03.-04.03./08.03.-11.03.

21.04.-28.04.2018

06. 10.-13.10.2018

03.11.-10.11.2018

\section{Kurs 4}

10.02.-17.02.2018

08.09.-15.09. 2018

\section{Kurs 5}

26.05.-02.06. 2018

01.12.-08.12.2018

\section{Refresher Woche}

12.03.-16.03.2018

Refresher Wochenende

17.11.-18.11.2018

\section{Sonderkurs}

Klinisches Taping Teil II 14.05.2018
Graz

Graz

KA1841

KA1842

$\begin{array}{ll}\text { Bad Häring } & \text { KA1831 } \\ \text { Graz } & \text { KA1832 } \\ \text { Graz } & \text { KA1833 } \\ \text { Wien } & \text { KA1834 }\end{array}$

Graz

KA1851

KA1852

St. Johann

KA1871

Loipersdorf

KA1861

Graz

KA1891

\section{Ausbildungsziel}

Das Erlernen von klinisch-manuellen Untersuchungstechniken am Stütz- und Bewegungssystem zur therapeutischen Beeinflussung von reversiblen Funktionsstörungen.

Die Fortbildung dient damit der Erweiterung des Angebotes der kurativen, rehabilitativen und präventiven Medizin.

\section{Zielgruppe}

ÄrztInnen für Allgemeinmedizin und FachärztInnen aller Sonderfächer.

\section{Teilnahmevoraussetzung}

Frühest möglicher Beginn der Fortbildung ist nach der Promotion.

\section{Weiterbildungsdauer/Stundenerfordernis}

Mindestens 1,5 Jahre, nach den Richtlinien der Österreichischen Ärztekammer sind für das ÖÄK Diplom Manuelle Medizin insgesamt 300 Fortbildungsstunden (100 Stunden Theorie, 200 Stunden Praxis und Demonstrationen) erforderlich.

\section{Wesentliche Inhalte}

Erkennen und Behandeln mit den Händen

Von der Arbeitshypothese zur Diagnose

Prävention

Rezidivprophylaxe

Terminänderungen/Absagen vorbehalten.

Wir weisen darauf hin, dass für die Kursteilnehmer der ÖAMM die Kurszeiten und Anwesenheitspflicht bindend sind!

Die Bestätigung der Fortbildungspunkte durch die österreichische Ärztekammer ist an die Erfüllung der Kursrichtlinien gebunden.

Verbindliche Anmeldungen und Auskünfte im Sekretariat der ÖAMM

E-Mail: fortbildung@oamm-graz.at

Internet: www.oamm-graz.at

Adresse: Kärntner Straße 419, 8054 Graz

Tel. \& Fax: +43 (0) 316-283 483 